\title{
Factors influencing rehabilitation outcomes in prelingually deafened late implanted cochlear implant users and development of a
} nomogram

\author{
Riyuan $\mathrm{Liu}^{1}$, Qian WANG ${ }^{2}$, Qingshan $\mathrm{Jiao}^{3}$, Fei $\mathrm{Ji}^{1}$, hui zhao ${ }^{1}$, Jianan $\mathrm{Li}^{3}$, and SM Yang ${ }^{4}$ \\ ${ }^{1}$ Chinese PLA General Hospital \\ ${ }^{2}$ Military General Hospital of Beijing PLA \\ ${ }^{3}$ General Hospital of People's Liberation Army \\ ${ }^{4}$ Department of Otolaryngology Head and Neck Surgery, Institute of Otolaryngology, \\ Chinese PLA General Hospital,
}

April 4, 2021

\begin{abstract}
Objectives: Our study aimed to identify potential factors that influence rehabilitation outcomes in late-implanted adolescents and adults with prelingual deafness and attempted to develop a reliable nomogram. Design: This retrospective study included 120 subjects less than 30 years of age who had received cochlear implantation at a single medical center. The Categories of Auditory Performance (CAP) scale was used to evaluate the rehabilitation outcomes. A nomogram was constructed using the $\mathrm{R}$ and EmpowerStats software. Results: Univariate analysis indicated higher rates of auditory performance improvement in younger aged subjects. Residual hearing and regular and longer implant use were more common among subjects showing auditory performance improvement. Multivariate analysis identified residual hearing (Hazard Ratio, 6.11; 95\% Confidence Interval, 1.83-20.41; $\mathrm{P}<0.01$ ), age at implantation (Hazard Ratio, 0.31; 95\% Confidence Interval, 0.14-0.83; $\mathrm{P}=0.02$ ) and regular CI use (Hazard Ratio, 7.79; 95\% Confidence Interval, 2.50-24.20; P<0.01) as independent predictors for auditory performance improvement. The nomogram's predictive performance was satisfactory as verified by the calibration curve and Receiver operating characteristic (ROC) curve. Conclusions: We demonstrated that residual hearing, younger age, and regular CI use were associated with auditory performance improvement in this cochlear implant user population. Our nomogram model demonstrated satisfactory predictive performance for this population.
\end{abstract}

Factors influencing rehabilitation outcomes inprelingually deafened late implanted cochlear implant users and development of a nomogram

Abstract

Objectives: Our study aimed to identify potential factors that influence rehabilitation outcomes in lateimplanted adolescents and adults with prelingual deafness and attempted to develop a reliable nomogram. Design: This retrospective study included 120 subjects less than 30 years of age who had received cochlear implantation at a single medical center. The Categories of Auditory Performance (CAP) scale was used to evaluate the rehabilitation outcomes. A nomogram was constructed using the R and EmpowerStats software. Results: Univariate analysis indicated higher rates of auditory performance improvement in younger aged subjects. Residual hearing and regular and longer implant use were more common among subjects showing auditory performance improvement. Multivariate analysis identified residual hearing (Hazard Ratio, 6.11; 95\% Confidence Interval, 1.83-20.41; $\mathrm{P}<0.01$ ), age at implantation (Hazard Ratio, 0.31; 95\% Confidence Interval, 0.14-0.83; $\mathrm{P}=0.02$ ) and regular CI use (Hazard Ratio, 7.79; 95\% Confidence Interval, 2.50-24.20; 
$\mathrm{P}<0.01)$ as independent predictors for auditory performance improvement. The nomogram's predictive performance was satisfactory as verified by the calibration curve and Receiver operating characteristic (ROC) curve.Conclusions: We demonstrated that residual hearing, younger age, and regular CI use were associated with auditory performance improvement in this cochlear implant user population. Our nomogram model demonstrated satisfactory predictive performance for this population.

\section{Key points:}

This study included 120 late-implanted cochlear implantation users with prelingual deafness, the most extensive sample size research in China so far.

In this retrospective study, $97(81 \%)$ exhibited rehabilitation benefits assessed by CAP scores before and after implantation, and 23 showed no perceivable improvement.

We developed and validated an easy-to-use nomogram to illustrate a model for predicting rehabilitation benefits after cochlear implantation.

The novel nomogram included three elements: age, residual hearing and regular cochlear implant use.

The predictive performance of the nomogram was satisfactory, as verified by the calibration curve and ROC curve.

Keywords : cochlear implant, prelingually deafened late implanted cochlear implant user, nomogram, rehabilitation

\section{Introduction}

The Second National Sample Survey on Disability in China reported 27.8 million people with hearing loss in $2006{ }^{1}$. More than half of a million people are $<18$ years of age, and 6.77 million are 18-60 years old. Although cochlear implants, as a sophisticated device for auditory stimulation, have been introduced into China for more than 20 years, everyone has yet to accept them as a medical device. It continues to present a financial challenge for many due to potentially prohibitive prices. As a result, some prelingually-deaf individuals become late-implanted users. This particular cochlear implant user population in China is yet to be studied.

Previous results in the literature both refute and support late cochlear implantation in prelingually deaf adults. Poor speech discrimination is reported firstly, which may be related to long-term auditory deprivation and lack of auditory memory. Some encouraging results have been reported as surgery techniques and cochlear implant technologies have improved, including improving speech recognition and quality of life after cochlear implantation. Some scholars have attempted to summarize potential predictive factors for functional benefits, such as age at implantation, communication mode, residual hearing, education, and so on. However, results from one study are not consistently confirmed by other studies, probably for reasons such as characteristics of the study population, variable inclusion and evaluation criteria across medical centers, and relatively small subject sample sizes ${ }^{2}$.

To mitigate the impacts mentioned above, we collected a relatively large sample $(n=120)$ from our medical center. We aimed to assess rehabilitation outcomes in late-implanted prelingually deafened adolescents and adults, identify predictive factors, and develop a nomogram incorporating CI to predict rehabilitation outcomes.

\section{Methods}

\subsection{Participants}

Data of patients aged 7 to 30 years who received cochlear implants at the *** Hospital, Beijing, China, between January 1, 2003, and December 31, 2017, were retrospectively analyzed. All subjects had finished a series of preoperative audiological and imaging studies, including tympanometry, auditory brainstem responses, temporal bone computerized tomography, and magnetic resonance imaging. The subjects were 
selected based on the following criteria: binaural severe to profound hearing loss with onset before the age of 3 years, late implantation (at 6 years or older), realistic expectations, and willingness to participate in follow-up visits.

Medical records were reviewed for gender, age at diagnosis of sensorineural hearing loss (SNHL), age at implantation, residence address, body mass index (BMI), side of the operation, usage of hearing aid, and preoperative hearing tests. Information on rehabilitation training, the CI use pattern (regularly or not), and the time of CI use were obtained during follow-up visits. Our study defined regular CI use at [?]8 hours per day. According to the implantation age, subjects were divided into 3 groups: $<10$ years, $10-20$ years, and 20 - 30 years. To gauge the subject's living environment, the residence address was used to classify the set into three categories: rural, small town, and metropolitan. Based on the age at diagnosis of SNHL and caregivers' medical history, deafness was classified as congenital or acquired. Based on hearing test results, subjects were categorized as having complete deafness (pure tone threshold $>120 \mathrm{~dB}$ HL or ASSR threshold $>120 \mathrm{~dB}$ nHL) or residual hearing (hearing threshold [?]120 dB HL).

\subsection{Categories of Auditory Performance (CAP) Scales}

The CAP, developed by Archbold, was used to rate auditory ability before and after cochlear implantation ${ }^{3}$. The score was categorized into eight levels from 0 to 7 (Table1). Two audiologists collected and scored CAP data in a face-to-face fashion. Another supervising audiologist would review the assessment to resolve any disagreements. Subjects were divided into two groups based on therapeutic outcomes assessed by CAP scores: "improved" (post-operative score better than pre-operative score) and "ineffective" (no improvement in CAP score or subject refusing to use the implant).

\subsection{Statistical Analysis}

Statistical analyses were conducted using the SPSS vision 25 software (IBM, Armonk, NY). Single-factor analyses were done first. Student's t test or Rank sum test (Mann-Whitney U test) was performed to detect differences in body mass index and time of CI use between the improved and ineffective groups. Chisquare tests were used for categorical variables, such as gender, age group, address, side of the operation, classification of deafness, use of hearing aid, residual hearing, and rehabilitation training. A P-value of $<0.05$ was considered statistically significant. Multivariate analysis was done secondly. Logistics regression analysis was used in this step. Receiver operating characteristic (ROC) analysis was used to illustrate model performance (larger area under the curve, AUC, indicating more accurate predictions). Hosmer-Lemeshow test was used to test the goodness of fit for the logistic model ( $\mathrm{P}>0.05$, no evidence of lack of fit.).

\subsection{Nomogram}

Based on logistic regression analysis, a nomogram model was developed using the "rms" package in the $\mathrm{R}$ Version 2.14.1 (http://www.R-project.org) and EmpowerStats (X\&Y Solutions Inc, Boston MA) software. Validation of the nomogram was assessed by discrimination and calibration. The ROC curve evaluated discrimination of the nomogram by comparing nomogram-predicted vs. observed probability. The calibration curve was derived based on regression analysis, and 500 bootstraps resamples were applied to the activities.

\section{Results}

\subsection{Patient Characteristics and Follow-up}

Of the 120 subjects with prelingual deafness (who received cochlear implantation at our medical center) included in this retrospective study, 97 (81\%) exhibited rehabilitation benefits assessed by CAP scores before and after implantation, and 23 showed no perceivable improvement. Before implantation, the effective group socred $1.1 \pm 1.0$ and the ineffective group scored $0.9 \pm 1.1$. During follow-up visit, the effecvive socred $5.3 \pm 1.5$ and the ineffective group scored $0.9 \pm 1.1$. The rate of effectiveness gradually dropped along with age increase and was $94.3 \%$ for patients under 10 years old, $80.3 \%$ for those between 10 and 20 years old, and $57.9 \%$ for those between 20 and 30 years old, respectively $(\mathrm{P}<0.01)$. The residual hearing was present in $87.6 \%$ of patients demonstrating improvements and in only $52.2 \%$ of those without improvements $(\mathrm{P}<0.01)$. Patients 
showing improvements also reported more regular cochlear implant use than those without improvements (77.3\% vs. $30.4 \%, \mathrm{P}<0.01)$ with longer $\mathrm{CI}$ usage time $(5.7 \pm 3.0$ vs. $3.6 \pm 4.5$ years, $\mathrm{P}<0.05)$. No statistically significant difference $(\mathrm{P}>0.05)$ was identified for gender, BMI, residence location, side of the operation, history of deafness, use of hearing AID, or structured rehabilitation training (Table 2).

There was no distinct correlation between residual hearing and regular $\mathrm{CI}$ use $(\mathrm{r}=0.12, \mathrm{P}=0.18)$, nor between residual hearing and age $(\mathrm{r}=-0.13, \mathrm{P}=0.15)$ or between regular $\mathrm{CI}$ use and age $(\mathrm{r}=-0.14, \mathrm{P}=0.13)$.

\subsection{Predictive factors}

Multivariate analysis showed that residual hearing (Hazard Ratio, 6.11; 95\% Confidence Interval, 1.83-20.41; $\mathrm{P}<0.01$ ), age at implantation (Hazard Ratio, 0.31; 95\% Confidence Interval, 0.14-0.83; $\mathrm{P}=0.02$ ) and regular CI use (Hazard Ratio, 7.79; 95\% Confidence Interval, 2.50-24.20; $\mathrm{P}<0.01$ ) were independent predictors for improvement. Time of CI use $(\mathrm{P}=0.08)$ was a non-significant factor, as the outcome of logistics regression analysis showed.

Omnibus Tests of Model Coefficients confirmed a difference, indicating statistically significant differences between at least two tested parameters $(\mathrm{P}<0.01)$. Hosmer-Lemeshow test showed the goodness of fit for the logistic model $\left(\chi^{2}=0.53, \mathrm{P}=0.97\right)$. The area under the ROC curve was 0.84 (95\% Confidence Interval, 0.74-0.94), indicating a satisfactory accuracy.

\subsection{Nomogram}

The novel nomogram incorporating the predictive factors (Figure1) illustrated that age at implantation and regular CI use shared the largest contribution to prediction, followed by residual hearing. The nomogram can be used in a few steps: firstly take the points for each factor, then summarize the total points, then predict the value and probability. For example, a patient at 11(38 points) with preoperative residual hearing (84 points) who uses CI regularly (92.5 points) will have a total score of 214.5 points, and the probability of benefits is $>90 \%$.

The calibration curve of the nomogram is depicted in Figure 2 and ROC in Figure 3. The AUC was 0.859 (95\% Confidence Interval, 0.761-0.957, sensitivity $=94.9 \%$, specificity $=69.6 \%$, accuracy $=90.0 \%$, positive predictive value $=92.9 \%$, negative predictive Value $=76.2 \%$, positive likelihood ratio $=3.12$, negative likelihood ratio $=0.07$, diagnostic odds ratio $=42.05$ ).

\section{Discussion}

To our knowledge, this study included the largest number of late-implanted adolescents and young adults with prelingual deafness at a single medical center in China to this date. What's more, multivariate analysis in the present study illustrated three independent factors predicting rehabilitation results: i.e., residual hearing, age group, and regular CI use. A simple-to-use nomogram model was developed for late-implanted adolescents and young adults ( $<30$ years old) with prelingual deafness, with satisfactory prediction performance.

Residual hearing is regarded as an essential predictor as it may offer acoustic stimulus for the development and maintenance of central auditory pathways. Some scholars have reported positive effects of residual hearing. For example, Carlson reported a 63-percentage-point improvement of open-set speech recognition in pediatric implantees with residual hearing ${ }^{4}$. Gordon demonstrated that children with more residual hearing showed higher speech perception scores over the first year of implant use than children with poorer hearing ${ }^{5}$. A more recent study showed the same ${ }^{6}$. Consistent with these positive findings, our results also showed that residual hearing was an independent predictor correlated with rehabilitation benefits in our lateimplanted prelingual deafened young patient population. However, we could not illustrate the mechanism directly, although it is possible, as mentioned above, that residual hearing provided some acoustic stimulation benefitting the development and maintenance of auditory cortical centers and central auditory representation integrity ${ }^{7}$. Of course, this mechanism needs to be further investigated.

Our study showed an inverse correlation between age and post-implantation auditory performance improvement. The rate of benefits dropped from $94.3 \%$ to $57.9 \%$ as age increased from under 10 to between 20 and 
30. For those younger subjects who got used to implant usage, it can be inferred that their central auditory system remained relatively functional. Other scholars have also stressed the importance of age. Harrison used three different speech recognition tasks to evaluated 82 congenitally deaf children ranging from 1.9 to 15.4 years old ${ }^{8}$. He reported that the age of operation was a more prominent factor when using complex measurement. There were hypotheses such as reduced plasticity, delayed development of frameworks involved in communication, and decoupling of auditory pathways.

For postoperative factors, our multivariate analysis demonstrated the correlation between rehabilitation outcomes and regular use of cochlear implants, with regular CI use found more commonly in patients showing benefits than those without benefits. Our study defined regular CI use at [?]8 hours per day. Regular CI use involves two elements: i.e., time and frequency, both of which essential for adaption and practice. Likewise, Nava revealed that some sound localization ability could emerge in the condition of a long time and extensive CI use for late implanted adults ${ }^{9}$. As Strelinikov indicated in a review of PET neuroimaging articles, reorganization of the brain functional architecture needed a long-term period ${ }^{10}$. Regular CI use is necessary for good practice to restore auditory cognitive processing in implanted subjects.

Nomograms have been widely used in cancer research, especially for predicting disease, for example, survival in patients with resected lung cancer or recurrence of hepatic carcinoma ${ }^{11,12}$. Some cardiologists and radiologists expanded nomogram usage to predict survival in coronary artery calcium in recent years ${ }^{13}$. However, no nomogram can predict the effect of CI. We developed and validated an easy-to-use nomogram to illustrate a model for predicting rehabilitation benefits after cochlear implantation. This novel nomogram contains three routinely collected clinical variables and should be easy to understand for both patients and caregivers during the consultation. Both otologists and patients can estimate the probability of benefits after cochlear implantation using this scoring system.

This study had several limitations. First, the sample size was not sufficiently large in our study, which might lead to statistical biases and reduce results' reliability. Second, our study used a subjective measurement, although two audiologists were involved in scoring to ensure objectivity. Third, our study's rate of improvement cannot be directly compared with other studies using different measurements, such as closed-set auditory discrimination and open-set word recognition. The results of the CAP can be affected to some extent by the ceiling effect. Therefore, an objective measurement with a detailed classification or pointscoring system needs to be used in future studies. Fourth, the real-world application of this nomogram to late-implanted prelingually deafened adults remains to be determined.

In conclusion, to our knowledge, this study demonstrated for the first time that age, residual hearing, and regular CI use are associated with rehabilitation benefits in late-implanted young patients with prelingual deafness through multivariate logic analysis. The proposed nomogram based on statistical analysis is a novel and easy-to-use tool for otologists and late-implanted patients less than 30 years old.

\section{References}

1 Li JN, Chen S, Zhai L, et al. The Advances in Hearing Rehabilitation and Cochlear Implants in China. Ear Hear. 2017. 38(6): 647-652.

2 Teoh SW, Pisoni DB, Miyamoto RT. Cochlear implantation in adults with prelingual deafness. Part I. Clinical results. Laryngoscope. 2004. 114(9): 1536-1540.

3 Archbold S, Lutman ME, Marshall DH. Categories of Auditory Performance. Ann Otol Rhinol Laryngol Suppl. 1995. 166: 312-314.

4 Carlson ML, Sladen DP, Haynes DS, et al. Evidence for the expansion of pediatric cochlear implant candidacy. Otol Neurotol. 2015. 36(1): 43-50.

5 Gordon KA, Twitchell KA, Papsin BC, Harrison RV. Effect of residual hearing prior to cochlear implantation on speech perception in children. J Otolaryngol. 2001. 30(4): 216-223. 
6 Lammers M, Versnel H, Topsakal V, van Zanten GA, Grolman W. Predicting Performance and Non-Use in Prelingually Deaf and Late-Implanted Cochlear Implant Users. Otol Neurotol. 2018. 39(6): e436-e442.

7 Chiossi J, Hyppolito MA. Effects of residual hearing on cochlear implant outcomes in children: A systematic-review. Int J Pediatr Otorhinolaryngol. 2017. 100: 119-127.

8 Harrison RV, Gordon KA, Mount RJ. Is there a critical period for cochlear implantation in congenitally deaf children? Analyses of hearing and speech perception performance after implantation. Dev Psychobiol. 2005. 46(3): 252-261.

9 Nava E, Bottari D, Bonfioli F, Beltrame MA, Pavani F. Spatial hearing with a single cochlear implant in late-implanted adults. Hear Res. 2009. 255(1-2): 91-98.

10 Strelnikov K, Marx M, Lagleyre S, Fraysse B, Deguine O, Barone P. PET-imaging of brain plasticity after cochlear implantation. Hear Res. 2015. 322: 180-187.

11 Wang Y, Li J, Xia Y, et al. Prognostic nomogram for intrahepatic cholangiocarcinoma after partial hepatectomy. J Clin Oncol. 2013. 31(9): 1188-1195.

12 Liang W, Zhang L, Jiang G, et al. Development and validation of a nomogram for predicting survival in patients with resected non-small-cell lung cancer. J Clin Oncol. 2015. 33(8): 861-869.

13 Ó Hartaigh B, Gransar H, Callister T, et al. Development and Validation of a Simple-to-Use Nomogram for Predicting 5-, 10-, and 15-Year Survival in Asymptomatic Adults Undergoing Coronary Artery Calcium Scoring. JACC Cardiovasc Imaging. 2018. 11(3): 450-458.

\section{Hosted file}

Figures.pdf available at https://authorea.com/users/405784/articles/516643-factorsinfluencing-rehabilitation-outcomes-in-prelingually-deafened-late-implanted-cochlearimplant-users-and-development-of-a-nomogram

\section{Hosted file}

Tables.pdf available at https://authorea.com/users/405784/articles/516643-factorsinfluencing-rehabilitation-outcomes-in-prelingually-deafened-late-implanted-cochlearimplant-users-and-development-of-a-nomogram 